\title{
The S.A.F. Double Star Section's Contribution to the INCA-HIPPARCHOS Programme
}

\author{
$P$. Durand
}

Ecole Marcel Sembat, F-18000 Bourges,France

The Double Star Section of the Société Astronomique de France has been in existence since 1980. It has set up observing groups covering various areas: close binaries, photographic measurements, re-observation of neglected pairs, and astrolabe measurements. All this has been thanks to our sceintific advisors, professionals specializing in double stars: Muller, Couteau, Soulié, Bacchus, and Dommanget. The Section meets twice a year, and it was at Lille in 1984 that J. Dommanget suggested that we should collaborate in the programme of identifying doubtful doubles for the input catalogue for the future satellite Hipparcos.

The first observations were made after the meeting in November 1984, from Paris and with amateur equipment, thanks to an initial list prepared by Prof. Bacchus. After several months we could tell that our equipment was insufficient, and that photocopies of the Palomar Sky Survey were not accurate enough. We needed more powerful instruments and a different organisation to resolve the ambiguity in identification that can arise by two close stars being confused and given the same coordinates, by the presence of a cluster, or by the existence of a faint star that is not given in the catalogues. In all these cases, identifying the star means being able to give it a designation, allowing it to be found on an atlas, and obtaining better coordinates so that the ambiguity is removed. It is a question of astrometry.

In November 1985, the decision was taken to request three allocations of telescope time for the summer of 1986: at Nice (50-cm refractor), Observatoire de Haute-Provence ( $80-\mathrm{cm}$ reflector), and Pic du Midi (1-m reflector). Eight to ten nights were allocated to us on each telecsope. The 4 observers were equipped with the AAVSO, Papadopoulos and PSS Atlases, and fifty-odd forms, which permitted the field to be identified on a PSS plate by $x$ and $y$ rectangular coordinates, gave the equatorial coordinates and details of the double required for identification purposes. A chronometer allowed the position to be related to reference stars by using the $\Delta \alpha$ and $\Delta \delta$ differences, from the known size of the instrument's field. On site, the teams planned their observations, arranging them in order of increasing RA, and distribtuion throughout the night, and familiarizing themselves with an observatory telescope that was new to them by establishing the fields of the various eyepieces.

Work at the eyepiece consisted of setting the telescope to the given coordinates, corrected for precession, checking the finder field against the AAVSO chart, centring the object, recognizing the main field with the help of the PSS, examining the double 
at a power of 350 to 600 in order to identify it, and making a sketch. The next day, in the office, the double was located on the PSS by using the observation, and new $\left(x^{\prime}, y^{\prime}\right)$ coordinates were established. The completed forms were sent to Prof. Bacchus for the identification to be checked and for more precise coordinates to be calculated, possibly with reference to a catalogue.

Table 1. Results of three 1986 programmes

\begin{tabular}{llllll}
\hline Observations & \multicolumn{4}{l}{ Identifications } \\
Place & planned & carried out & confirmed & doubtful & Not seen \\
\hline Nice & 69 & 51 & 44 & 2 & 5 \\
OHP & 51 & 48 & 35 & 5 & 9 \\
Pic & 43 & 28 & 23 & 5 & 0 \\
\hline
\end{tabular}

Not all the identifications apparent to observers were retained. We have found that what appeared to be a simple problem hid actual complexity on the sky. It is not just by chance that these doubles have been little observed and have remained poorly identified.

This amateur/professional collaboration has enabled us to develop and allowed us to contribute in the area where we are most at home: that of observation. It has also taught us to work more rigorously, and more methodically, thanks to advice from Prof. Bacchus. Finally, it has also revealed some neglected pairs that are in motion and which ought to be measured accurately. It has also been a step towards other programmes, in particular it has laid the foundation for the success of the 1987 campaign, three trips to Nice and the Pic du Midi, which we hope will give as good results as those we obtained in 1986. 